\title{
Crystal structure of rat transthyretin at 2.5 Å resolution: First report on a unique tetrameric structure*
}

\author{
Andrzej Wojtczak \\ Faculty of Chemistry, N. Copernicus University, J. Gagarina 7, 87-100 Toruń, Poland
}

Key words: rat transthyretin, prealbumin, crystal structure

\begin{abstract}
The first observation of a unique tetrameric molecular structure of transthyretin from rat (rTTR, prealbumin) is reported. The structure has been determined by $X$-ray diffraction using molecular replacement and the structure of human transthyretin (hTTR) as a starting model. Crystals of native rat

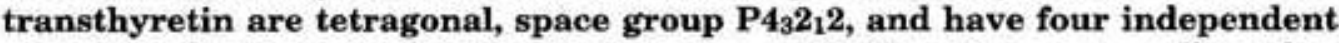
monomers in the asymmetric unit of the crystal lattice. Data were collected to $2.5 A$ resolution and the structure has been refined to $R=18.9 \%$ for 13584 data points between 8-2.5 $\AA$ resolution. Like hTTR, the rat protein is also a $54000 \mathrm{Da}$ tetramer with four identical polypeptide chains of 127 amino-acid residues. Of the 22 amino-acid residues which are different in the human and rat TTR sequences, none are in the thyroxine binding domain. Analysis of these data reveal that the tertiary structure of rTTR is similar to that of hTTR with only small differences in the flexible loop regions on the surface of the protein. As a result of local changes in flexible loop regions near residues $30-41,60-65$ and 102-104, the structure of rTTR monomers is more compact than that of the corresponding hTTR monomers. The loop between residues $30-41$ is bound closer to the monomer core in the former as compared with the latter structure and there is a wider opening of the space formed between these loops at two adjacent monomeric subunits. These conformational changes do not affect the interfaces between the monomeric subunits and are not transmitted to the thyroxine binding site so that its topology remains not altered.
\end{abstract}

In man, transthyretin (hTTR, prealbumin), is one of three serum proteins which transports thyroid hormones through the general circulation and is responsible for binding about $20 \%$ of the circulating thyroxine $\left(3,5,3^{\prime}, 5^{\prime}\right.$-tetraiodo-L-thyronine; T4). It also binds retinol-binding protein which carries vitamin $A$ [1]. In lower vertebrates such as rat, transthyretin is the primary serum thyroid hormone transport protein and thus has a greater physiological role than it does in man [2]. Rat transthyretin (rTTR) has $85 \%$ sequence homology to the human protein and has 22 amino-acid changes in the 127 amino-acid residue monomers [3, 4] (Table 1). Most of the sequence differences occur in peripheral loop regions while the hormone binding domain is strictly conserved.

As previously reported, all structures of human TTR crystallize in the orthorhombic space group $\mathrm{P} 2{ }_{1} 2_{1} 2$ with two independent monomeric $\beta$-barrels ( $A$ and $B$ ) in the asymmetric unit of the crystal lattice [5-12]. Also chicken transthyretin crystallizes in a hex-

*This work was supported in part by the State Committee for Scientific Research Grants 6/P203/005/05 and 6/P04A/032/11 and a Fogarty FIRCA grant TW00226.

Abbreviations: FAP, familial amyloid polyneuropathy; SA, simulated annealing; hTTR and rTTR, human and rat transthyretin, respectively. 
Table 1. Sequence comparison between human $(h)$ and rat $(r)$ transthyretin and human amyloidcausing variants (a) which are coincident in locations to the seven sequence changes in rat TTR.

Residues of the finger-like loop are underlined. The residues directly involved in thyroxine binding interactions in human TTR are marked with the bold lettering.

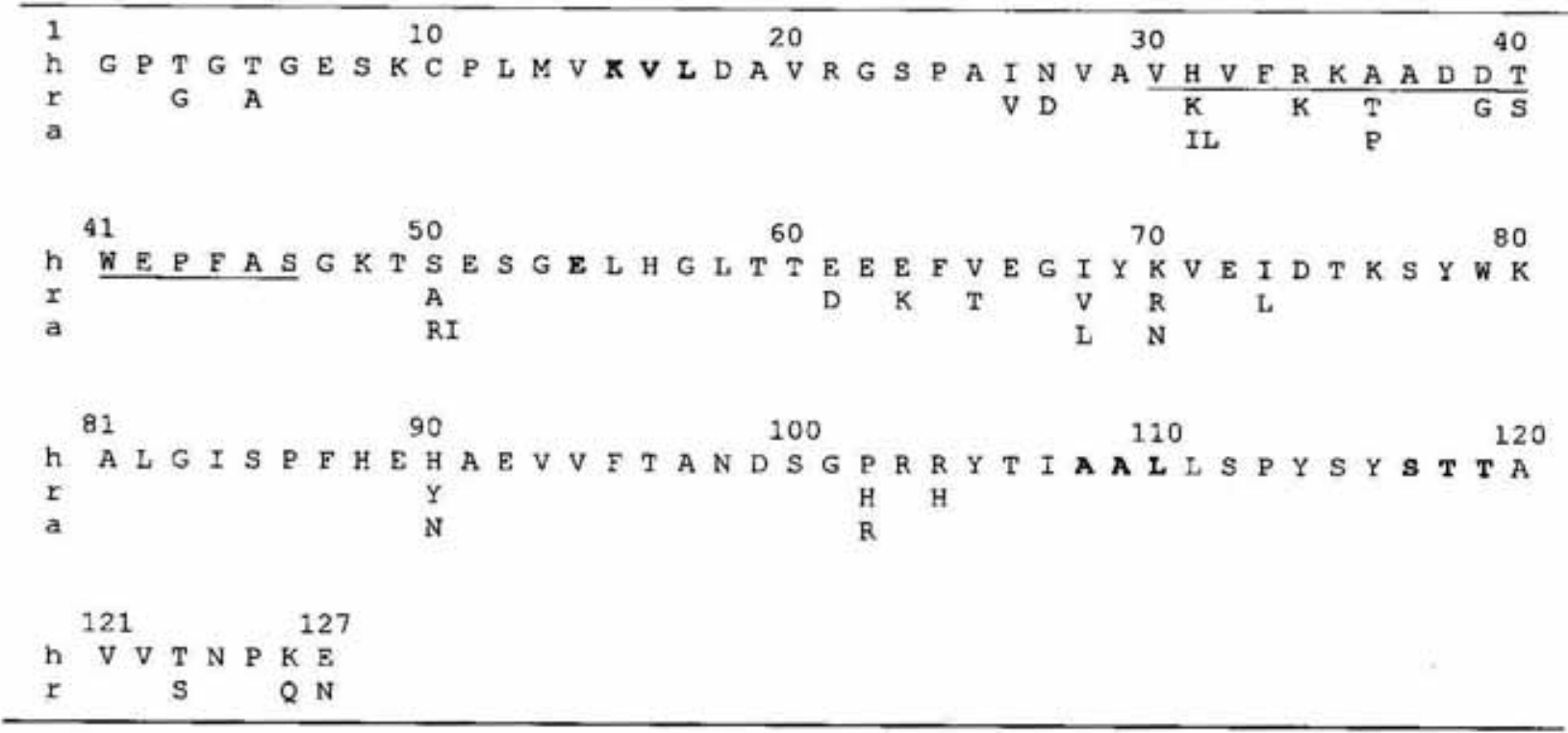

agonal space group with only two subunits in the asymmetric unit [13]. These monomers are assembled around a central channel such that the tetramer has molecular 222 symmetry. The crystallographic [001] axis is coincident with the channel axis and results in twofold symmetry of the ligand binding site. The two hormone binding sites per tetramer are formed by pairs of twofold related monomers (A-A' and B- $\mathrm{B}^{\prime}$ ) positioned across the channel axis. The independent monomers $A$ and B are further related by pseudo twofold symmetry axis parallel to the crystallographic [010] axis. In the case of ligandbound hTTR complexes, interpretation of the ligand binding interactions has been hampered by the presence of twofold averaged electron density in the channel representing the two symmetry related orientations of the bound ligand. Therefore, all reported human transthyretin ligand complexes show a $50 \%$ disorder in the occupation of the binding domain $[7,8,14,15]$.

The aim of this research was to study the influence of the 22 sequence differences between human and rat TTR on the intermolecular interactions and to determine those structural features which influence crystallization of human TTR as a unique dimer rather than the functional tetramer.

\section{EXPERIMENTAL METHODS}

Pooled rat sera were purified using a modification of the human transthyretin protocol, as described in [11]. Unliganded rat TTR was crystallized from $55-65 \%$ ammonium sulfate, $0.1 \mathrm{M}$ acetate buffer, $\mathrm{pH} 5.0$, using the hanging drop technique with HANGMAN $[16,17]$. Diffraction data with 16548 unique reflections to $2.5 \AA$ resolution were collected from a $0.2 \mathrm{~mm} \times 0.2 \mathrm{~mm} \times 0.6 \mathrm{~mm}$ crystal using a Rigaku R-axis II imaging plate system and an R-200 rotating anode generator. The average intensity/sigma ratio was 6.0 for all data, the cumulative data percentage was $82.0 \%$, and the Rmerge for these data was $10.4 \%$. The crystals are tetragonal, space group $\mathrm{P}_{3} 2_{1} 2$, with cell dimensions of a $=82.73 \AA$ and $\mathrm{c}=161.80 \AA$ and a complete tetramer in the asymmetric unit.

The structure of rTTR was determined by molecular replacement procedures within the program XPLOR [18], using the protein coordinates for the tetramer from hTTR-3, $3^{\prime}-\mathrm{T}_{2}[8]$ as a starting model. Examination of the self-rotation function for rTTR revealed non-crystallographic 222 molecular symmetry, as expected from hTTR structures. The cross-rotation function solution was determined and the translation functions search 
followed by Patterson Correlation was performed in all space groups corresponding to the point group 422 for data between $12-4 \AA$ resolution. The translation and packing function in XPLOR indicated a correct solution in the space group $\mathrm{P}_{3} 2_{1} 2$. Rigid body refinement of this solution confirmed the space group assignment of $\mathrm{P}_{3}{ }_{3} 2$ and resulted in $\mathrm{R}=26.6 \%$ for data between $10-3.5 \AA$ resolution. The $R$ index was reduced to $18 \%$ following simulated annealing (SA) and slow cooling protocols [18]. During the SA and conventional refinement, no constraints accounting for the non-crystallographic symmetry were applied. The asymmetric unit contains a complete tetramer of rTTR. The model was verified by calculating a series of residue-deleted electron density maps using CHAIN [19] on a Silicon Graphics Elan system. During the refinement, the set of programs written by G.D. Smith [20] was used. Final refinement was carried out using XPLOR [18] for 13584 data $F>3 \sigma(F)$ of a resolution gradually extended to $2.5 \AA$ and resulted in $\mathrm{R}=18.9 \%\left(\mathrm{R}_{\text {free }}=30.3 \%\right.$ for 1532 reflections). The structural model consists of residues 8-127 for each monomer. The attempts to locate the missing residues 1-7 failed because of poorly defined electron density. After the examination of difference electron density maps, 130 water molecules, positioned closer to the protein polar groups than the sum of atomic van der Waals radii, were placed in the structure. The conformational statistics show that $77.2 \%$ of the residues are positioned in the most favoured regions of the Ramachandran plot (Fig. 1) as calculated with the program PROCHECK [21]. The superposition of the tetramers of rTTR on hTTR reveals little conformational difference in their $\beta$-sheet structures (Fig. 2), while there are some differences in the loop regions between residues 59-65 and 98-105 of all monomers. The best model for these loops was built with the omit map analysis. The possible multiple conformations in these fragments and the missing residues $1-7$ in all subunits result in the relatively high $R_{\text {free }}$ value and affect the ratio of residues found in the most favoured conformation on Ramachandran plot. The final refinement statistics is summarized in Table 2. The co- ordinates of rTTR have been deposited with the Protein Data Bank (access code 1GKE).

\section{RESULTS}

The four monomeric subunits of rat TTR constitute the asymmetric part of the crystal structure. The A and B monomers of rTTR are equivalent to hTTR unique $\mathrm{A}$ and $\mathrm{B}$ subunits, rTTR subunits $\mathrm{C}$ and $\mathrm{D}$ correspond to human TTR A' and ' ' $^{\prime}$ monomers, related in the human structure to A and B by a crystallographic twofold axis and therefore characterized by identical geometry and revealing identical interactions. Slight differences have been found in the geometry of the four subunits of rTTR, reflecting different intermolecular interactions due to crystal packing. As expected, the major differences in main chain conformation between the subunits are found in flexible regions $34-41$ and 98-104, both revealing some disorder in all known crystal structures of human and rat transthyretin.

Rat TTR shares $85 \%$ sequence homology with the human protein. Most of the sequence differences occur in peripheral loop regions, while the thyroid hormone binding domain is strictly conserved (Table 1). Therefore, comparison of the overall geometry of transthyretin from rat and human, based on an r.m.s. fit of the backbone atoms [20], reveals high similarity between both proteins (Fig. 2). Some of the sequence differences have little effect on the local geometry of the TTR monomeric subunits. The major conformational changes are due to multiple substitutions between the protein sequences, the majority of which are located in the strand-turn-strand fragments between residues $30-41$, and the loop regions of residues 55-72 (Fig. 3). These substitutions result in the slight size differences between rat and human TTR monomeric subunits.

In monomer A of hTTR, the His-31 side chain is H-bonded to the carboxylic group of Glu-42 from a symmetry-related tetramer, with an N...O contacts of $2.8 \AA$, while in subunit B His-31 interacts with the carboxylic group of Glu-72 via a water molecule. In the rat protein subunit A, Lys-31 forms a 


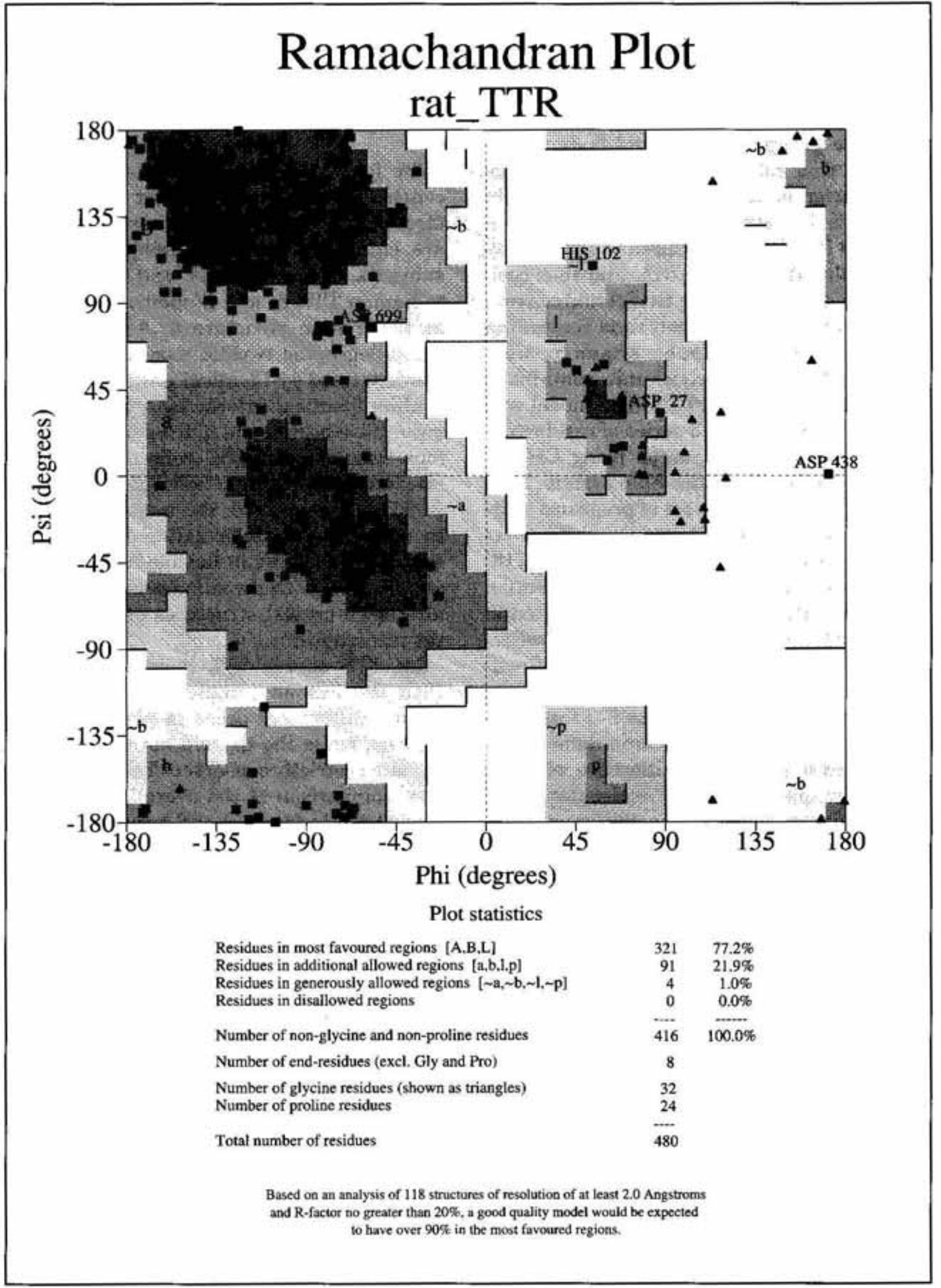

Figure 1. The Ramachandran plot for rTTR refined to $2.5 \AA$ (prepared with PROCHECK [21]). Glycine residues are shown as triangles and all other residues as squares. 


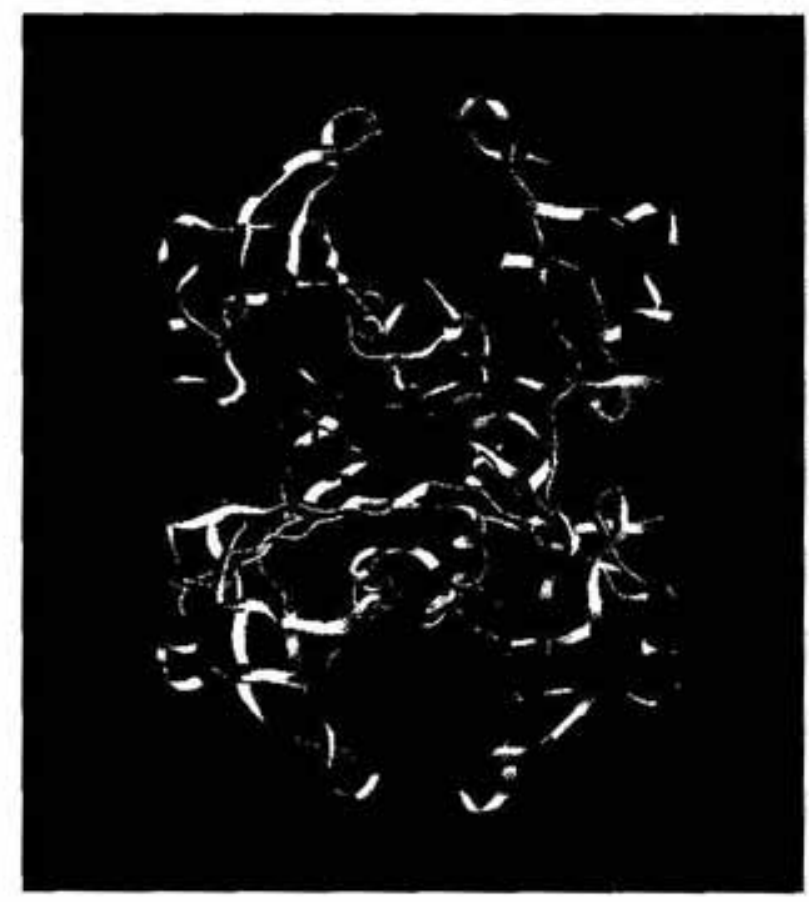

direct interaction (N...O 3.0 Å) with Glu-42 of a symmetry-related tetramer (Fig. 4 ; contact $31 . .642$ ), while in subunit $D$ it interacts (N...O, 3.5 $\AA$ ) with the hydroxyl of Ser-40 and carbonyl of Trp-41 of a symmetry-related tetramer (Fig. 4; contact 631...40). In monomers B and C the Lys-31 do not form any intermolecular interaction with the adjacent TTR molecule (Fig. 5ab).

There are four substitutions in the loop region encompassing residues 34-40. In hTTR residues Arg-34, Ala-36, Asp-39 and

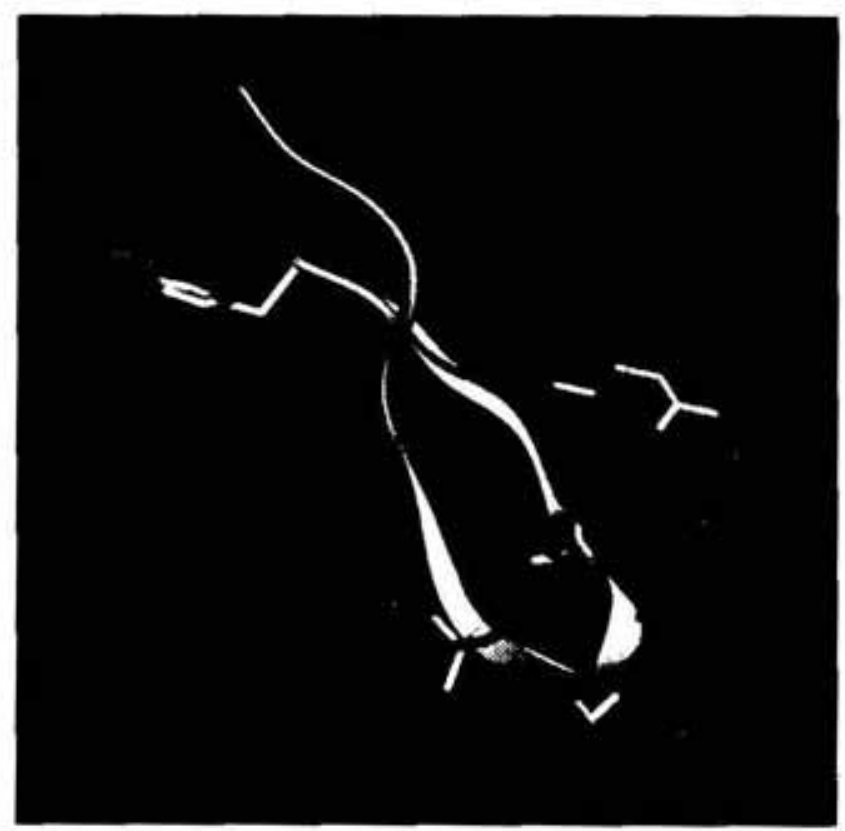

Figure 2. Comparison of the alpha carbon representation, made with the program SETOR [22], of rat TTR (magenta) and the human TTR-T 4 complex (yellow).

Also shown is the position of thyroxine (green) in the binding sites. The TTR tetramers were superimposed by least-squares program PROFIT [20].

Thr- 40 are replaced by Lys, Thr, Gly and Ser, respectively, in rTTR (Fig. 3 ). The r.m.s. fit of the backbone atoms for the human and rat TTR tetramers was performed with PROFIT program [20]. As illustrated for subunit D (Fig. 3), the maximum shift for backbone $\mathrm{C} \alpha$ atoms between loops is $2.6 \AA$ at residue 39 , the r.m.s. difference being $0.83 \AA$. The analogous values for subunits $\mathrm{A}, \mathrm{B}, \mathrm{C}$ and D are $1.6 \AA, 2.0 \AA$ and $1.4 \AA$, respectively. For comparison, the r.m.s. fit of the main chain atoms of rTTR monomers has revealed dif-
Figure 3. Comparison of the flexible loop segment between residues $30-49$ made with the program SETOR [22] for human (yellow) and rat (magenta) TTR.

Shown are the side chains for those residues that differ between the two proteins. Superposition with PROFIT [20]. 
ferences not exceeding $1.75 \AA$ for the loop $\mathrm{C} \alpha$ atoms positions, with the r.m.s. differences less than $0.87 \AA$. The relatively high r.m.s. values for the superposition are strongly affected by the contribution of 98-105 loop atoms in all monomers, with the main chain atom shifts as high as $5 \AA$, while for most atoms this value does not exceed $0.4 \AA$.

These loop residues $30-41$ reveal different interactions in human and rat TTR. In human transthyretin Arg-34 is exposed on the loop surface, Ala-36 side chain does not form any specific interaction. The rat TTR loop is more tight due to the Thr-36 forming $\mathrm{H}$ bonds with the adjacent amino acids over the loop tip. The Lys-34 is buried between the monomeric core residues Phe-63, Thr-65 and Tyr-69. As a result of these interactions the

Table 2. Refinement statistics for the structure of rat TTR.

\begin{tabular}{lc}
\hline Resolution range $(\AA)$ & $8.0-2.5$ \\
Reflections observed & 16584 \\
\% observed & 82.0 \\
Reflections used $(3 \sigma(\mathrm{F}))$ & 15116 \\
R factor (13584 refl.) & 0.189 \\
R free (1532 refl.) & 0.303 \\
Protein atoms & 3696 \\
Water molecules & 130 \\
B factor (Wilson stat.) $\left(\AA^{2}\right)$ & 11.61 \\
B factor (average) $\left(\AA^{2}\right)$ & 14.18 \\
B factor (protein) $\left(\AA^{2}\right)$ & 14.07 \\
B factor (water) $\left(\AA^{2}\right)$ & 17.33 \\
r.m.s. deviations from ideality: & \\
Bond distance r.m.s. $(\AA)$ & \\
Angle r.m.s. $\left({ }^{\circ}\right)$ & 0.010 \\
Improper angle r.m.s. $\left({ }^{\circ}\right)$ & 1.838 \\
Ramachandran plot & 1.441 \\
Residues in disallowed regions & $77.2 \%$ \\
\hline Puzzati plot $(\AA)$ & 0.25 \\
\hline & \\
\hline &
\end{tabular}

loop structure is positioned slightly closer to the monomeric barrel core, and the cylindrical space between the loops of the rTTR monomers is opened wider than found in hTTR.

The largest change in this loop region is a consequence of the replacement of the negatively charged Asp side chain at position 39 of hTTR with Gly in rTTR (Fig. 3). In hTTR, either the carboxylic group of Asp-39 or its carbonyl oxygen interacted with Lys-35. In the rat TTR monomer B the space corresponding to the hypothetical position of the missing Asp carboxylic group is occupied by Gly-639 of a symmetry related tetramer, although no specific polar interaction is formed. In hTTR Lys-35 formed the intramolecular interaction to Asp-39. In subunits $A$ and $C$ of rTTR, Lys-35 does not participate in any polar interactions. However, in monomer B, the Lys-35 and Asp-38 from the adjacent tetramers form a pair of interactions (N...O 2.6 $\AA, 2.8 \AA$ ), (Fig. 4; contacts $235 . .638$, $238 \ldots 635$ ). Due to the crystal packing in rTTR, only the monomer D hydroxyl group of Ser- 40 forms the intermolecular $\mathrm{H}$-bond with Lys-31 of an adjacent monomer (Fig. 4; contact $40 . .631$ ).

The largest r.m.s. differences in the polypeptide chain atom positions (up to $5 \AA$ ) are found in the 98-105 loop region with multiple sequence differences, which folds near the helical turn residues $61-63$. In hTTR, this loop has different backbone conformations in subunits $A$ and $B$ such that there is a $180^{\circ}$ flip of the backbone between anchoring residues 100 and 103 . This suggests that there is significant flexibility in this region of TTR [7-9]. Rat TTR shows similar changes in this region and the conformations in subunits $\mathrm{A}$ and $\mathrm{C}$ are similar to subunit A in hTTR, while subunits B and D to hTTR subunit B. The consequence of these alternate conformations is demonstrated by differences in their intermolecular interactions with neighboring residues. In hTTR Glu-61 is exposed to solvent. The rat Asp-61 forms close contacts His-102 and His-104 in subunits $\mathrm{A}$ and $\mathrm{C}$, and has contacts to Arg103 and His-104 in subunits B and D. The net result is a greater complementarity in the 


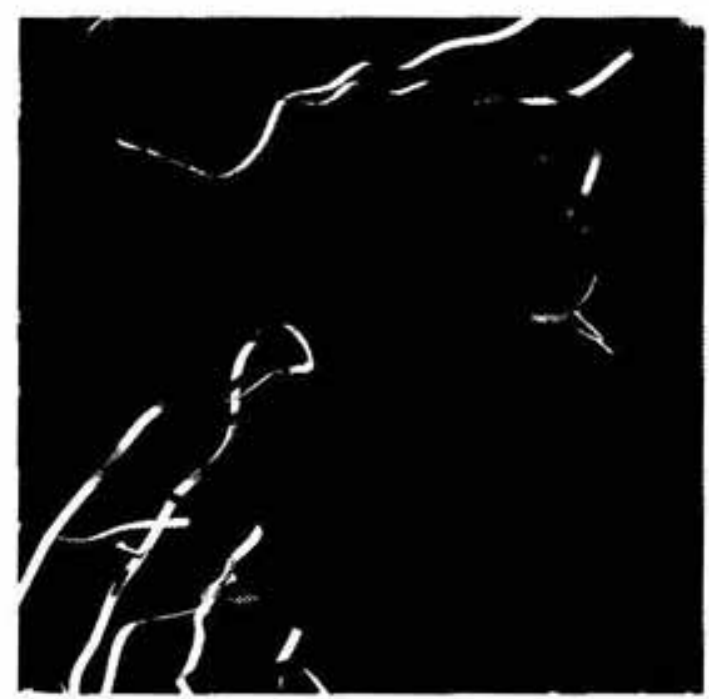

rat sequence in these neighboring polypeptide chains to optimize their electrostatic interaction, an environment not found in the human sequences in this region.

As a result of these local changes between flexible loops in the rat structure, the monomeric subunits are more compact than in human TTR structures. This is confirmed by measurements of the size of the $\beta$-barrel of each monomer and by measurements of the distances across the flexible loops of adjacent monomers (Table 3 ). The distance across the external face of each $\beta$-barrel is measured between the $\mathrm{C} \alpha$ atom of residue 37 and 100 and in the finger-shaped flexible loop (residues $30-49$ ) between residues 37 and 40 (Fig. 3). These data reveal that the $\beta$-barrel of the rat protein is more compact and that the flexible loops are positioned closer to the core of the tetramer.

\section{Comparison of crystal packing of rat and human TTR}

The predominant topological features of a transthyretin tetramer are the finger-like structures built up with residues $30-45$ of each monomeric subunit. Pairs of these strand-loop-strand structures form the cylindrical cavities at the top and bottom of the tetramer (Fig. 2). The side surfaces of the TTR tetramers are shaped with the residues following the last turn of the monomers $\alpha$-helix (residues 81-83) and loop fragments with residues $98-103$ of each monomer. There is a shallow cleft over the interface between the
Figure 4. Illustration of fragment $30-49$ packing interactions in rat TTR made with the program SETOR [22].

The sequence numbers and color for subunits $\mathrm{A}, \mathrm{C}$ and symmetry-related B, D are: A (white) residues 8-127, C (yellow) residues 408-527, and B (green) residues $208-327$ and $D$ (magenta) residues 608-727. Hydrogen bonded interactions are highlighted (red) between Lys31 and Glu-642, Ser-40 and Lys-631, Glu-235 and Asp-638, Asp-238 and Lys-635, and between Pro-243 and Asp-438.

four monomeric subunits, formed between these loops on each side of the tetramer and limited by the C-terminal fragments of the polypeptide chains. The bottom of the cleft is formed by the $\beta$-turn fragments containing residues $19-22$ and $50-53$. The $\mathrm{N}$-terminal and C-terminal fragments of each subunit are positioned on the tetramer side surface near the channel entrance. Most of the amino acids differing between human and rat TTR sequence are found in these fragments, clearly affecting the intermolecular interactions and crystal packing.

The location of amino acids differing between rat and human TTR sequences can be described in four general groups. The first group consists of Leu-73, the amino acid with the side chain buried in the monomeric $\beta$-barrel of TTR. The substitution I73L does not affect the protein structure since the side chain of residue 73 is located in the hydrophobic environment of the monomeric $\beta$-barrel, where is enough space to accommodate either of the two amino acids. In both structures the side chain forms hydrophobic interaction with Ala-109 methyl group.

The second series consists of a number of substitutions (from human to rat TTR) that occur at the cleft on the side surface of the tetramer. This series includes substitutions near the tetramer center (S50A, I26V and N27D), as well as the loop region substitutions near the channel entrance (E61D, E63K, V65T, P102H and $\mathrm{R} 104 \mathrm{H}$ ) and the C-terminal substitutions (T123S, K126Q, E127N). These modifications make some 

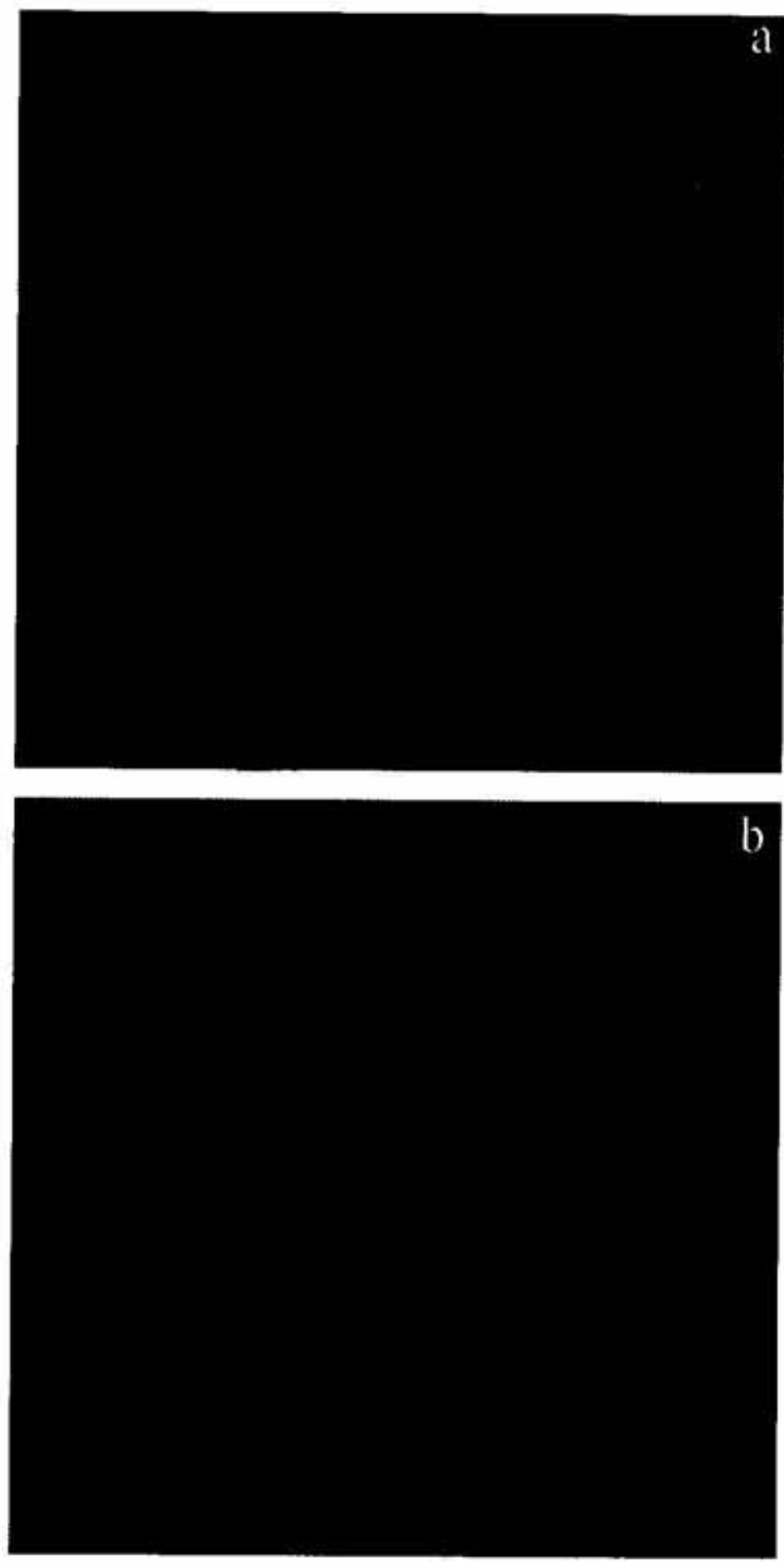

Figure 5. 2Fo-Fe electron density map contoured at $1 \sigma$ level revealing differences in the interactions of Lys-31 in rTTR (drawn with CHAIN [19]): (a) intermolecular interactions with symmetry related Glu-642 (orange) and intramolecular interactions with Glu-72 in monomer A; (b) cluster of water molecules (red crosses) around the ammonium NZ group of Lys-431 in monomer C, mediating intramolecular interactions with Glu-472.

more space available deep in the cleft in rTTR and result in an increase of the hydrophobicity of the cleft surface and an increase of the space available for penetration of adjacent tetramer side chains, while the charges near the entrance to the tetramer channel vanish in rTTR.

The third group of substitutions take place deep in the cavity between the finger-like loops at the top and bottom of the tetramer
(Fig. 2), near the monomer-monomer interface. I68V in the lower $\beta$-sheet structure and the H90Y substitution result in an increased hydrophobicity of the surface and an enlargement of the space available for conformational adjustment between TTR subunits in rat. The K70R substitution results in an increased accessibility of the positively charged side chain for the intermolecular interactions. 


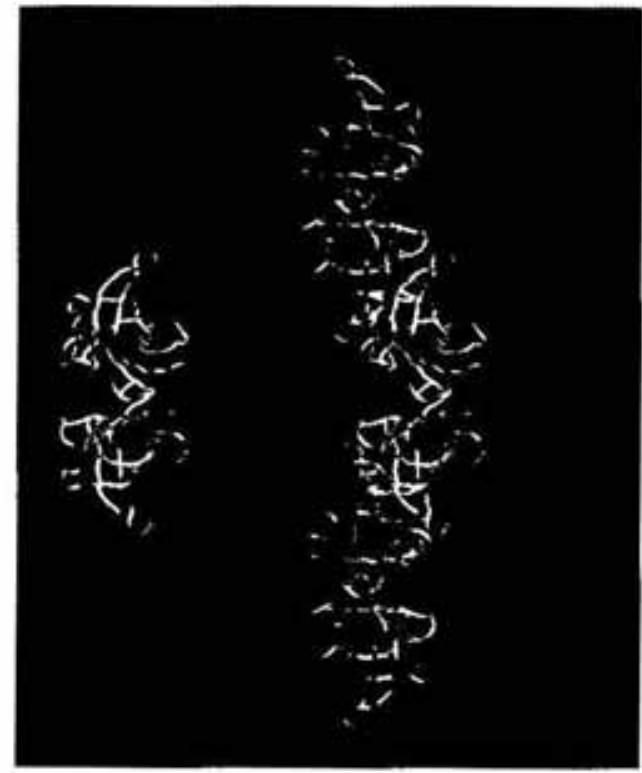

The fourth group of substitutions occurs in the finger-like loop 30-45. The external loop surface contains R34K near the monomeric barrel core and A36T. In hTTR the guanidinium moiety of Arg-34 is exposed on the protein surface. In rat TTR Lys-34 side chain is buried between polar amino acids in the monomeric core, altering the local electrostatics of the surface, when compared to hTTR. In rTTR the Thr-36 hydroxyl group forms a series of $\mathrm{H}$-bonds tightening the loop. The D39G substitution creates the gap over the loop structure filled with the Lys-35 side chain in rat TTR, while in human TTR Asp39 formed a salt bridge to Lys- 35 . The T50S substitution enhances the capabilities of polar interaction formation at the loop basis. This series of substitutions enhances local hydrophobicity of the loop surface in rat TTR, strongly affecting the packing interactions in the crystal lattice.

The human TTR tetramers form the layers parallel to crystallographic (100) plane, with intra-layer packing interactions engaging the external surfaces of the $30-45$ strandloop-strand fragments (Fig. 6). The layer contains some spaces filled with the next layer $(n+1)$ translated along the [011] diagonal. The inter-layer interactions are established between layers $n$ and $(n+2)$ and involve the loop residues following the $\alpha$-helices on both side surfaces of the TTR tetramer. Channels parallel to the crystallographic $z$ axis are formed between TTR tetramers in the crystal
Figure 6. Crystal packing of hTTR in the space group $P 22_{12} 2$ projected along the $z$ axis (drawn with SETOR [22]).

The tip of the flexible loop between residues $30-49$ is highlighted at residue 38 . The color pattern reflects the individual monomeric subunits which generate the complete tetramer in the crystal lattice.

lattice and filled with solvent molecules. This arrangement of the TTR molecules in the lattice results in the orthorhombic $\mathrm{P} 2{ }_{1} 2_{1} 2$ symmetry.

Inspection of the crystal packing in the tetragonal space group $\mathrm{P}_{3} 2_{1} 2$ reveals that the closest intermolecular contacts for rat TTR differ from those observed for the human structure (Figs. 6, 7) $[8,9]$. In the rat structure, the close contacts are found between residue Gly-57 of subunit $C$ (subunit $\mathrm{A}^{\prime}$ in the human structure) and its symmetry related molecule. The tetramer is rotated with respect to the twofold axis along the diagonal of the tetragonal lattice such that there are no close contacts among the other monomers (Fig. 7). This is in contrast to the packing observed in the orthorhombic lattice of hTTR in which there are close monomermonomer contacts between the independent B monomer and its symmetry related copy. These contacts involve loops between residues $40-45$ and $60-67$ (Fig. 6; loop regions marked with 38 ).

In rat TTR the crystal lattice is formed from tetramer layers that are perpendicular to the crystallographic $z$ axis (Fig. 7) with interactions involving both internal and external surfaces of the 30-45 strand-loop-strand fragments. The fragments from A and B monomers form a four-loop connection with the $\mathrm{C}$ and $\mathrm{D}$ monomers of the symmetry related tetramer, and the tip of the subunit B loop is positioned between similar frag- 


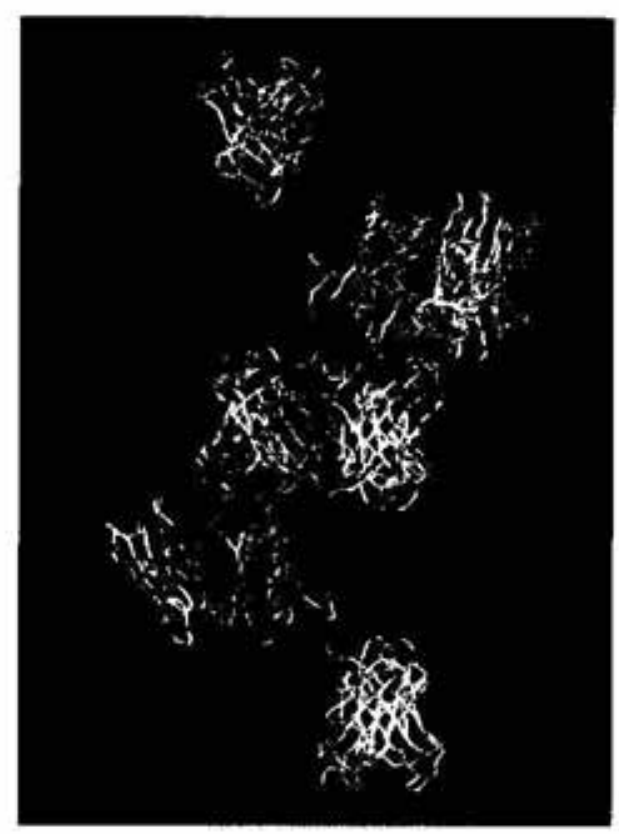

ments in subunits C and D of another TTR tetramer (Figs. 4 and 7). The layers are also assembled via interactions involving foursubunit interfaces on the side surfaces of the tetramers, but these interactions differ from those found in the human TTR structure. These non-equivalent interactions result in a different number of contacts shorter than $3.5 \AA$. The adjacent layer is rotated $90^{\circ}$ with respect to the crystallographic $z$ axis and results in a series of contacts along the surface of the tetramer (Fig. 7). While in hTTR the tetramers interacted only with the residues following the last turn of the $\alpha$-helix, in rTTR these residues penetrate the cavities between analogous fragments and $\beta$-turn fragments 111-114 on the adjacent tetramer, reaching deeper towards the 19-22 $\beta$-turn residues at the bottom of the cavity. The number of close contacts are 14,25, 43 and 36 , respectively for monomers A, B, C and D of rTTR.

\section{DISCUSSION}

The crystal structure of rat TTR, which crystallizes in the tetragonal space group $\mathrm{P}_{3} 2_{1} 2$, reveals for the first time the details of a unique tetrameric transthyretin structure. In addition, the 22 residue changes in the sequence between rat and human
Figure 7. Crystal packing of rTTR in the space group $P 4{ }_{3} 212$ projected along the [110] diagonal (drawn with SETOR [22]).

Each independent monomer is colored: A (white), B (green), C (yellow), and D (magenta).

transthyretin allow to study the effects of residue changes on structure. The analysis of these data reveal that, although the overall tertiary structure of rat TTR is similar to that of human TTR, the conformational changes brought about by the 22 amino-acid differences favour crystallization of the complete tetramer in the crystal lattice. As illustrated in the packing diagram of rTTR (Fig. 7), the orientation of the rat tetramer is such that it does not lie along any crystallographic symmetry element as is the case for the human structure. Although the tetramer has molecular 222 symmetry, the four monomers are structurally independent. In the human structure, which is aligned along the $z$ axis of the lattice, the closest intermolecular contacts are more extensive and involve two flexible loop regions encompassing residues $40-46$ and $60-65$.

As a result of five residue substitutions in the loop between residues $30-41$, this loop is positioned closer to the protein core than observed in the hTTR structures. These conformational changes open a cavity between the loops from adjacent monomers at the top and bottom of the molecule. The shift of both loop regions affects adjacent regions of the molecule - i.e., there is a more extensive network of interactions with residues 50-70. Furthermore, the latter fragment transmits these conformational changes to the loop re- 
gion between-residues 97-103. Residues $30-42$ and $55-72$ form numerous interactions, resulting in a more extensive network of side chain-side chain contacts. For example, the side chains of Lys-35 and Trp-41 penetrate deeper into the pocket between Val-68 and Lys-70 of rat TTR than found in the analogous region of hTTR. Also Lys-34 interacts with residues 63 to 69 . The shift of loops $30-42$ and $55-72$ is also transmitted to the surface loop 98-104, moving it closer to the tetramer channel entrance. The result of these shifts is tighter binding of the loop $30-41$ toward the monomer core, and a wider opening of the space formed between these loops of two adjacent TTR monomers (Fig. 2). Consequently, the rat TTR is more compact, and has more side chain-side chain interactions in the surface loop regions, than the human protein. This is supported by the distances measured across the external face of each monomeric $\beta$-barrel (Table 3 ). These differences, as well as the constraints of molecular symmetry of the two crystal lattices, are reflected in their crystal packing. The conformational changes do not affect the interfaces between the monomeric subunits, and are not transmitted to the thyroxine binding site which does not alter its topology. The determination of crystal structures of some TTR variants related to the Familial Amyloid Polyneuropathy (FAP) [9, 10, 12] had not revealed any significant structural differences between amyloid-causing variants and the wild-type hTTR. There might be several different mechanisms of the fiber formation depending on the point mutation in a specific TTR variant. The reason for the amyloid deposition might be the decreased tetramer stability due to partial unfolding of the monomeric $\beta$ barrels. Blake and coworkers (cf. Terry et al. [10]) hypothesized that in the Met-30 variant of hTTR the slight conformational change, leading to disulfide bridge formation by Cys-10, might be a reason for the fiber formation. There are seven positions in the polypeptide chain of TTR common to rat TTR, human wild-type TTR and its FAP variants. Among known FAP variants involving mutations in those positions, seven out of nine are characterized by substitutions increasing the hydrophobicity of the side chain (Table 1). In both human and rat transthyretin the regions of amino acids known to be FAP related are involved in intermolecular interactions. A new hypothesis might be proposed, that these point mutations, by enhancing local hydrophobicity of the tetramer surface, alter the intermolecular interactions favouring association of TTR tetramers followed by formation of insoluble associates and their deposition as fibers in the affected organs. From this point of view, the structure of rat transthyretin gives us an insight into the role of some surface amino acids in altering the intermolecular interactions of hTTR.

In summary, the observation of a tetrameric form of rat transthyretin provides the first opportunity to delineate unique monomer-monomer interactions of this thyroxinebinding protein and to define the role of

Table 3. Distances $(\AA)$ characterizing the monomeric $\beta$-barrel size in rat (rTTR) and human (hTTR) transthyretins

\begin{tabular}{|c|c|c|c|c|}
\hline & $\mathrm{C} \alpha-37$ & & $\mathrm{C} \alpha-37 \ldots \mathrm{C} \alpha-37$ & $\mathrm{C} \alpha-40 \ldots \mathrm{C} \alpha-40$ \\
\hline Subunit & & Subunit & & \\
\hline hTTR A & 19.79 & hTTR A-B & 25.35 & 11.77 \\
\hline hTTR B & 21.04 & & & \\
\hline rTTR A & 19.38 & rTTR A-B & 27.72 & 13.90 \\
\hline rTTR C & 18.03 & & & \\
\hline rTTR B & 18.65 & rTTR C-D & 27.74 & 14.31 \\
\hline rTTR D & 19.60 & & & \\
\hline
\end{tabular}


specific residues in ligand binding and tetramer formation, but also in tetramer intermolecular interactions. These differences can be exploited by comparison of the sites of the 22 substitutions between human and rat TTR.

The author wishes to thank Joseph R. Luft, Ilona Wawrzak and Colin Desnoes for their assistance in the purification of rat TTR and crystal growth experiments, Walter Pangborn for data collection, G. David Smith for the use of his programs, and Vivian Cody for useful discussions.

\section{REFERENCES}

1. Braverman, L.E. \& Utiger, R.D. (eds) (1991) The Thyroid, a Fundamental and Clinical Text; 6th edn., J.B. Lippincott, Philadelphia.

2. Robbins, J. (1991) Thyroid hormone transport proteins and the physiology of hormone binding; in The Thyroid, a Fundamental and Clinical Text; 6th edn. (Braverman, L.E. \& Utiger, R.D., eds.) pp. 111-125, J.B. Lippincott, Philadelphia.

3. Sundelin, J., Melhus, H., Das, S., Eriksson, U., Lind, P., Trägärdh, L., Peterson, P.A. \& Rask, L. (1987) The primary structure of rabbit and rat prealbumin and a comparison with the tertiary structure of human prealbumin. J. Biol. Chem. 260, 6481-6487.

4. Pettersson, T.M., Carlstrom, A., Ehrenburg, A. \& Jornval, H. (1989) Transthyretin microheterogeneity and thyroxine binding are influenced by non-amino acid components and glutathione constituents. Biochem. Biophysic. Res. Comm. 158, 341-347.

5. Blake, C.C.F. \& Oately, S.J. (1977) Protein-DNA and protein-hormone interactions in prealbumin: A model of the thyroid hormone nuclear receptor? Nature 268, 115-129.

6. De La Paz, P., Burridge, J.M., Oatley, S.J. \& Blake, C.C.F. (1992) Multiple modes of binding thyroid hormones and other iodothyronines to human plasma transthyretin; in The Design of Drugs to Macromolecular Targets (Beddell, C.R., ed.) pp. 119-172, J. Wiley \& Sons, Inc. New York.
7. Wojtczak, A., Luft, J.R. \& Cody, V. (1992) Mechanism of molecular recognition: Structural aspects of $3,3^{\prime}$-diiodo-L-thyronine binding to human serum transthyretin. J. Biol. Chem. 267, 353-357.

8. Wojtczak, A., Luft, J.R. \& Cody, V. (1993) Structural aspects of inotropic bipyridine binding: Crystal structure determination to $1.9 \AA$ of the human serum transthyretin-milrinone complex. J. Biol. Chem. 268, 6202-6206 .

9. Steinrauf, L.K., Hamilton, J.A., Braden, B.C., Murrell, J.R., \& Benson, M.D. (1993) X-ray crystal structure of the Ala-109-Thr variant of human TTR which produces euthyroid hyperthyroxinemia. J. Biol. Chem. 268, 24252430.

10. Terry, C.J., Damas, A.M., Oliveira, P., Saraiva, M.J.M., Alves, I.L., Costa, P.P., Matias, P.M., Sakaki, Y. \& Blake, C.C.F. (1993) Structure of Met 30 variant of transthyretin and its amyloidogenic implications. $E M B O$ J. 12, $735-741$.

11. Wojtczak, A., Cody, V., Luft, J.R. \& Pangborn, W. (1996) Ligand binding in human transthyretin complexes with thyroxine at 2.0 $\AA$ resolution and 3 ', $5^{\prime}$-dinitro- $N$-acetyl-L-thyronine at $2.2 \AA$ resolution. Acta Cryst. D52, 758-765.

12. Damas, A.M., Ribeiro, S., Lamzin, V.S., Palha, J.A. \& Saraiva, M.J. (1996) Structure of Val122Ile variant transthyretin - a cardiomyopatic mutant. Acta Cryst. D52, 966-972.

13. Sunde, M., Richardson, S.J., Chang, L., Pettersson, T.M., Schreiber, G. \& Blake, C.C.F (1996) The crystal structure of transthyretin from chicken. Eur. J. Biochem. 236, 491-499.

14. Cody, V., Wojtezak, A., Ciszak, E. \& Luft, J.R. (1991) Differences in inhibitor and substrate binding in transthyretin crystal complexes; in Progress in Thyroid Research (Gordon, A., Gross, J. \& Hennemann, G., eds.) pp. 793-796, Balema, Rotterdam.

15. Ciszak, E., Luft, J. \& Cody, V. (1992) Crystal structure determination at $2.3 \AA$ resolution of human transthyretin-3',5'- dibromo-2',4,4',6- 
tetrahydroaurone complex. Proc. Natl. Acad. Sci. U.S.A. 89, 6644-6648.

16. Luft, J.R., Cody, V. \& DeTitta, G.T. (1992) Experiences with HANGMAN: A macromolecular hanginig drop vapor diffusion technique. J. Crystal Growth 122, 181-185.

17. Luft, J.R. \& DeTitta, G.T. (1992) HANGMAN: A macromolecular hanging-drop vapor-diffusion technique. J. Appl. Cryst. 25, 324-325.

18. Brunger, A. (1992) XPLOR Version 3.1. A System for X-ray Crystallography and NMR. Yale University Press.
19. Sack, J.S. (1988) CHAIN: A crystallographic modeling program. J. Mol. Graphics 6, 224-225.

20. Smith, G.D. HWI Library of Programs.

21. Laskowski, R.A., MacArthur, M.W., Moss, D.S. \& Thornton, J.M. (1993) PROCHECK: A program to check the stereochemical quality of protein structures. J. Appl. Cryst. 26, 283-291.

22. Evans, S.V. (1993) SETOR: Hardware lighted three-dimensional solid model representations of macromolecules. J. Mol. Graphics 11, 148-153. 